\title{
Repeated or Continuous Medically Supervised Ketamine Administration Associated with Hepatobiliary Adverse Events: A Retrospective Case Series
}

\author{
Samantha Cotter ${ }^{1} \mathbb{D}$. Jennie Wong ${ }^{1} \cdot$ Neha Gada $^{1} \cdot$ Rajdeep Gill $^{1}$. S. Christopher Jones ${ }^{1} \cdot$ Grace Chai $^{1}$. \\ Daniel Foster ${ }^{2} \cdot$ Mark Avigan $^{1} \cdot$ Mallika Mundkur $^{1}$
}

Accepted: 12 September 2021 / Published online: 26 October 2021

This is a U.S. government work and not under copyright protection in the U.S.; foreign copyright protection may apply 2021

\begin{abstract}
Introduction Emerging off-label medical uses of ketamine for the treatment of persistent conditions such as depression and chronic pain often require repeated administration. Cases reported by other countries suggest that long-term and repeated exposure to ketamine may be associated with several risks, including but not limited to hepatobiliary damage.

Objective We aimed (1) to characterize the association between repeated administration of ketamine for off-label medical use and hepatobiliary events and (2) to describe recent trends in the use of ketamine across different clinical settings.

Methods We conducted a retrospective case series analysis, utilizing reports identified from the US Food and Drug Administration Adverse Event Reporting System database as well as the medical literature. We included all cases reported through July 2018 describing both repeated exposure to ketamine in a hospital or ambulatory setting and a hepatobiliary adverse event. We excluded cases describing ketamine abuse. We identified adverse hepatobiliary events using the Medical Dictionary for Regulatory Activities $\left(\right.$ MedDRA $^{\circledR}$ ) and summarized various case characteristics including: patient demographics, route of ketamine administration, dose, time to onset of event, type of event, and pre-existing risk factors for hepatobiliary disease. To assess trends in the demand for ketamine, we used IQVIA, National Sales Perspectives ${ }^{\mathrm{TM}}$ to provide the nationally estimated number of vials sold for ketamine from the manufacturer to all US channels of distribution from 2013 through 2017. Results We identified 14 unique cases that met selection criteria with 21 hepatobiliary adverse events including liver enzyme elevation in all cases, biliary dilation with liver cirrhosis $(n=1)$, biliary dilation with cholangitis $(n=1)$, and pericholeductal fibrosis $(n=1)$. Most cases received ketamine for the treatment of complex regional pain syndrome or chronic pain. In cases with a reported time to onset, the majority of events occurred within 4 days. The nationally estimated number of ketamine vials sold in the USA from manufacturers to various channels of distribution increased from 1.2 million in 2013 to 2.1 million in 2017.

Conclusions We report an association between repeated or continuous administration of ketamine and hepatobiliary adverse events. Increased awareness among clinicians may mitigate these adverse outcomes, especially in the context of growing ketamine sales.
\end{abstract}

Disclaimer: The views expressed are those of the authors and do not necessarily represent those of, nor imply endorsement from the US Food and Drug administration or the US Government

Samantha Cotter

Samantha.Cotter@fda.hhs.gov

1 Affiliated with the Office of Surveillance and Epidemiology, Office of Pharmacovigilance and Epidemiology, Center for Drug Evaluation and Research, US Food and Drug Administration, 10903 New Hampshire Ave, Silver Spring, MD 20993, USA

2 Affiliated with the Office of New Drugs, Center for Drug Evaluation and Research, US Food and Drug Administration, 10903 New Hampshire Ave, Silver Spring, MD 20993, USA

\section{Introduction}

Ketamine was originally approved by the US Food and Drug Administration (FDA) in 1970. Apart from the FDAapproved, single-use, facility-based indication of ketamine in anesthesia, it has been used off-label ${ }^{1}$ for several chronic outpatient diseases, such as treatment-resistant depression

\footnotetext{
1 The term "off-label" in the context of this paper is defined to include medically supervised or recommended use for a purpose other than the FDA-approved indication. The term "off-label" in this context does not include "abuse," "recreational use," or related terms, which refer to non-medical use in which ketamine is used for euphoric effects.
} 


\section{Key Points}

In this case series, we identified hepatobiliary adverse events occurring in association with repeated or continuous therapeutic administration of ketamine, which range in severity from asymptomatic elevation in liver enzymes to cholangitis and hepatic cirrhosis.

Clinicians should monitor patients for hepatobiliary events when prescribing or administering ketamine in recurrent doses for non-anesthetic indications.

and chronic pain syndromes [1-6]. A recently published survey of clinicians showed increasing use of ketamine for psychiatric conditions, with a variety of clinical specialties assuming responsibility for drug administration [7]. Although ketamine is approved for intravenous administration, off-label routes have included oral, sublingual, transmucosal, intranasal, subcutaneous, and intramuscular formulations [8]. Additionally, off-label use of ketamine spans multiple patterns of doses, frequencies, and durations of treatment. On 5 March, 2019, the FDA approved Spravato ${ }^{\circledR}$ (esketamine) nasal spray, the s-enantiomer of ketamine for use in adults with treatment-resistant depression in conjunction with an oral antidepressant [9].

There are studies of ketamine administration in depression and chronic pain; however, duration and characterization of short-term and long-term risks and benefits are not defined $[4,6,10,11]$. Repeat dosing of ketamine may be required to sustain a treatment response [12-14]. Clinical experts have voiced concern regarding increased clinically serious risks associated with repeated exposure to ketamine that include hepatobiliary adverse effects $[7,10,15,16]$. The medical literature describes hepatobiliary disease among individuals abusing ketamine in large doses and at high frequency. Multiple published case series suggest repeated recreational ketamine insufflation causes a cholestatic type of liver injury with biliary dilatation [17-22]. A precise quantification of the dose associated with these outcomes is complicated by variations in the insufflation technique and the purity of illicit drugs [22]. Further variation in the hepatotoxic risk of exposure to ketamine is linked to differences in the route of ketamine administration, with intranasal (8-45\%) and oral bioavailability (17-29\%) varying significantly compared with intravenous bioavailability (100\%) [23]. Additionally, there are reports in the literature of hepatobiliary injury associated with daily ketamine abuse [17, 19].

In addition, some regulatory authorities have raised safety concerns regarding the association between ketamine and hepatotoxicity, occurring in the context of medical use. In 2017, the French National Agency for the Safety of
Medicines and Health Products (ANSM) published a Dear Healthcare Professional Communication letter regarding the risk of severe liver damage during repeated and/or prolonged use of high-dose ketamine [24]. ANSM reported ten cases of serious liver damage (cholestatic cholangitis), including four cases requiring liver transplantation, since 2014, and recommended liver function test monitoring [24]. In response, the Malaysia's National Pharmaceutical Regulatory Agency issued an advisory to clinicians: "monitor patient's liver function closely if repeated and/or prolonged high doses of ketamine is used" [25].

In this analysis, we reviewed spontaneous report data submitted to the FDA together with cases reported in the published medical literature to identify cases that described a potential association between repeated or continuous administration of ketamine and hepatobiliary adverse events in the context of medical use. As a secondary objective, we sought to describe recent trends in the use of ketamine across different clinical settings within the USA.

\section{Methods}

\subsection{Data Sources}

To investigate the association between repeated ketamine exposure and hepatobiliary adverse events (AEs), we searched the FDA Adverse Event Reporting System (FAERS) and published literature for relevant cases through 30 July, 2018. FAERS, a database containing AE and medication error reports submitted to the FDA, is designed to support the FDA's post-marketing safety surveillance program for drug and therapeutic biologic products. The informatic structure of FAERS adheres to the international safety reporting guidance issued by the International Conference on Harmonisation (ICH E2B). Adverse events and medication errors are coded using Medical Dictionary for Regulatory Activities $\left(\right.$ MedDRA $\left.^{\circledR}\right)$ terminology [26].

We searched FAERS for reports describing the use of ketamine racemate, enantiomer, or metabolites (ketamine, esketamine, norketamine, $\mathrm{N}$-ethylnorketamine, and deschlorketamine). MedDRA ${ }^{\circledR}$ terms related to hepatobiliary AEs of interest included System Organ Class: Hepatobiliary Disorders; High Level Group Terms: Hepatic and Biliary Neoplasms Benign; and standardized MedDRA ${ }^{\circledR}$ queries related to biliary, hepatic, liver, gallbladder, and gallstone events (Table 1 of the Electronic Supplementary Material [ESM]). We searched published literature titles and abstracts using PubMed and Embase (Table 2 of the ESM). No language restrictions were applied in the search, and duplicates were identified through manual review. We screened all titles resulting from the search strategy to identify potential cases for inclusion, and the subset of titles identified as possibly 
relevant were used to guide a further review of abstracts and/ or full text if needed to determine eligibility for inclusion in the case series. All literature reports included ketamine and one or more of the following terms in PubMed (biliary, cholestasis, hepatic, hepatobiliary, cholangitis, hepatitis, or cholangiopathy) and Embase (hepatobiliary system, biliary tract disease, hyperbilirubinemia, cholestasis, obstructive jaundice, intrahepatic cholestasis, liver disease, sclerosing cholangitis, hepatitis, toxic hepatitis, or bile duct disease). Literature articles were further mined to identify additional relevant articles.

Among spontaneous case reports, only cases documenting hepatobiliary $\mathrm{AEs}^{2}$ in the context of repeated or shortterm continuous therapeutic ketamine administration were included. We considered ketamine use to be repeated in patients receiving more than one dose over a period extending beyond $24 \mathrm{~h}$. Cases from observational studies, retrospective analyses of hospital patient records [29], and randomized controlled trials were also included if hepatobiliary AEs were evaluated or reported in the context of off-label, medically supervised ketamine administration. We excluded reports indicating abuse, misuse, and overdose with ketamine, as well as reports of ketamine use in persons with a past medical history of abuse and misuse of ketamine or other products, owing to complexities of patients who abuse ketamine (i.e., long-term toxicities and disease-promoting effects of drug abuse including heart or lung disease, human immunodeficiency virus, viral hepatitis), allowing for a clearer drug-event relationship [30]. We also excluded reports of ketamine administration for its labeled indications (e.g., anesthesia for surgical procedures) and cases evaluated as "unassessable" or "unlikely" related to ketamine, according to the World Health Organization-Uppsala Monitoring Center causality assessment [31].

To assess the association between ketamine exposure and hepatobiliary AEs, extracted case information included sex, age, country of report, serious regulatory outcomes ${ }^{3}$, route of administration, reason for use, time to onset from ketamine exposure ${ }^{4}$, phenotypic characteristics of hepatobiliary

\footnotetext{
${ }^{2}$ When evaluating cases that reported laboratory values, the following levels were considered the upper limit of normal (ULN): alanine aminotransferase ULN $=40 \mathrm{U} / \mathrm{L}$, aspartate aminotransferase ULN $=$ $35 \mathrm{U} / \mathrm{L}$, ALP ULN $=115 \mathrm{U} / \mathrm{L}$, total bilirubin ULN $=1.2 \mathrm{mg} / \mathrm{dL}$ or $17.1 \mu \mathrm{mol} / \mathrm{L}$, gamma-glutamyl transferase $\mathrm{ULN}=85 \mathrm{U} / \mathrm{L}[27,28]$.

3 An AE or suspected adverse reaction is considered "serious" if, in the view of either the investigator or sponsor, it results in any of the following outcomes: death, a life-threatening $\mathrm{AE}$, inpatient hospitalization or prolongation of existing hospitalization, a persistent or significant incapacity or substantial disruption of the ability to conduct normal life functions, or a congenital anomaly/birth defect (Title 21 Code of Federal Regulations 314.80).

${ }^{4}$ When available, time-to-onset data included time following the start of ketamine and the cycle of therapy.
}

AE, dechallenge/rechallenge information, and clinical outcome(s) at the time of the report. As ketamine has been associated with different short-term, recurrent, and chronic forms of hepatobiliary injury, the causality assessments depended heavily on the temporal linkages between different patterns of ketamine exposure and the development of liver and bile duct abnormalities documented by imaging and/or biochemical diagnostic testing. When available, the measured effects of de-challenge and rechallenge with the agent were also evaluated. To accommodate the different reported patterns of ketamine exposure with both acute and chronic forms of liver toxicity, we used the WHO-UMC system for case-level causality assessment [31]. Our evaluation also incorporated diagnostic information when available to exclude alternative causes of acute liver injury when available, including viral hepatitis A, B, C, or E, cytomegalovirus, Epstein-Barr virus, pre-existing or autoimmune hepatitis, alcohol use, concomitant drugs with a National Institutes of Health Liver Tox Likelihood score [32] of A, B, or C, and past medical history of biliary disease including gallstones, cholecystitis, gallbladder disease, and cholangitis.

To understand trends in ketamine use, we used IQVIA National Sales Perspectives ${ }^{\mathrm{TM}}$ (NSP) to provide the nationally estimated number of ketamine vials sold from the manufacturer to all US channels of distribution ${ }^{5}$ from 2013 through 2017. Sales distribution data represent the amount of product sold from manufacturers to various settings of care and does not reflect what is being administered to patients directly. The amount of product purchased by various settings of care is a possible surrogate for use, assuming facilities purchase drugs in quantities reflective of actual patient use.

\section{Results}

\subsection{Hepatobiliary Events}

FAERS and literature searches retrieved 404 reports, and 1508 articles, respectively, from which 14 unique cases met our selection criteria (Fig. 1) [29, 33-38]. The selected cases occurred between 1995 and 2018, with eight cases reported between 2011 and 2018 (Table 1). Half of the individuals were female $(n=7)$ and half were male $(n=7)$. Reported ages ranged from 33 to 70 years (median 47.5 years). Most cases $(n=10)$ described parenteral ketamine administration. Reported reasons for ketamine use included treatment of

\footnotetext{
5 Various channels of distribution include retail (i.e., independent and chain pharmacies), non-retail (i.e., non-federal hospitals, federal facilities, clinics, long-term care), and mail order (i.e., standard and specialty mail).
} 


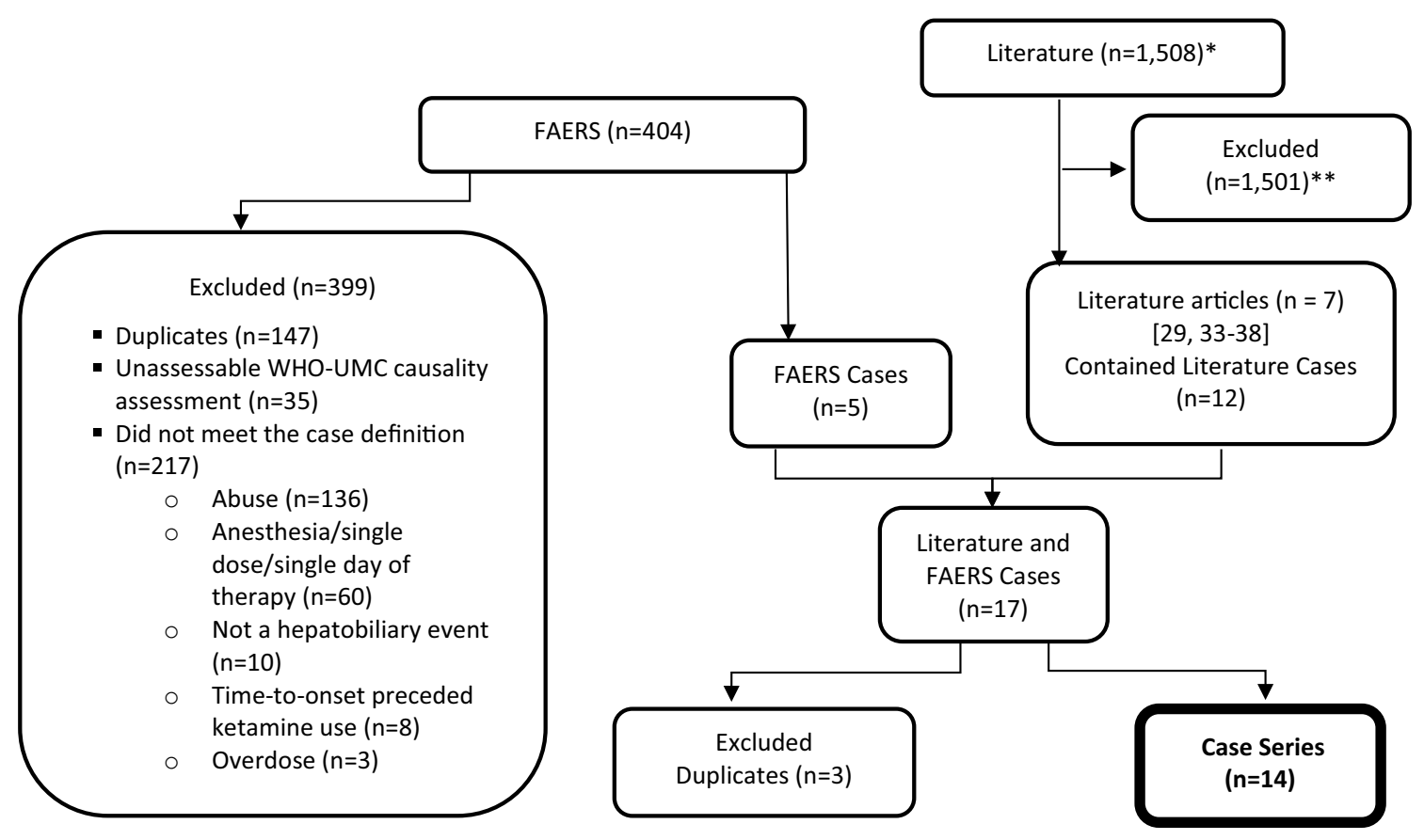

Fig. 1 Case selection (inclusions/exclusions). FAERS FDA Adverse Event Reporting System, WHO-UMC World Health Organization- Uppsala Monitoring Centre. *A total of 252 published articles or reports were identified with the first literature search strategy described in Methods, and a total of 1256 reports were identified with the second literature search strategy. **The majority of articles were excluded at the step of screening titles on the basis of not describing

complex regional pain syndrome $(n=9)$, postherpetic neuralgia $(n=2)$, chronic pain $(n=2)$, and chronic migraines $(n=1)$.

Dosage and route of administration of ketamine were variable. Of cases reporting dosing information $(n=13)$, regimens varied with respect to dose (weight-based dosing or fixed dose), route (oral and parenteral), rate, duration, and frequency (single dosages, weekly dosages, monthly dosages, or given over subsequent days). Some doses increased over a period of days or weeks, while other doses were fixed. Orally administered dosages ranged from 300 to $900 \mathrm{mg} /$ day in one patient and from 800 to $1600 \mathrm{mg}$ /day in the other patient. Intravenous dosage per hour $(n=9)$ ranged from 8 to $50 \mathrm{mg} / \mathrm{h}$ with a duration of 6 hours to 20 days. Some patients received repeat dosing; however, frequency was not always precisely reported.

We identified 14 unique cases ${ }^{6}$ representing 21 hepatobiliary $\mathrm{AEs}^{7}$. Based on WHO-UMC criteria, we assessed 12 cases as "probable" in their causal association with ketamine

\footnotetext{
${ }^{6}$ Unique case: patient who experienced hepatobiliary AEs on one or more occasions.

${ }^{7}$ Hepatobiliary AEs: hepatobiliary events including rechallenge events.
}

a clinical case report (e.g., reviews, basic science research, commentary). Other excluded articles did not meet the case definition (e.g., lack of the event of interest or described the development of hepatobiliary dysfunction in the context of ketamine abuse [i.e., not meeting the case definition applied for this study]) or were duplicates of cases identified from the first literature search or from cases identified from a search of the FAERS database

[31] (see Table 3 of the ESM). In cases with a reported time to onset, the majority of hepatobiliary events $(n=10)$ occurred within 4 days of ketamine exposure during cycle $1(n=2)$, cycle $2(n=5)$, cycle $3(n=2)$, and cycle $4(n$ $=1$ ) of administration (see Table 3 of the ESM). One case documented abnormalities 10 years following initial exposure [33]. All cases documented serum liver test elevations, including increases in gamma-glutamyl transferase $(n=8)$, alkaline phosphatase [ALP] $(n=8)$, and bilirubin $(n=5)$. In addition, we identified cases with elevated liver tests further complicated by biliary dilation, jaundice, liver cirrhosis, pericholeductal fibrosis, cholangitis, and cholangiopathy. Ketamine discontinuation was documented in 13 cases because of the onset of hepatobiliary events, of which all documented a positive dechallenge, and four unique cases later experienced a total of seven positive rechallenges. ${ }^{8}$ Documented risk factors included concomitant use of drugs independently associated with liver injury [32] $(n=4)$ and history of cholecystectomy $(n=2)$. Four cases provided enough detail to

\footnotetext{
8 Three cases experienced two positive rechallenges each and one case experienced one positive rechallenge for a total of seven positive rechallenges.
} 
Table 1 Descriptive characteristics of cases reporting hepatobiliary adverse events with ketamine in FAERS and the published medical literature through $30 \mathrm{July}, 2018(N=14)$

\begin{tabular}{|c|c|}
\hline \multicolumn{2}{|l|}{ Case source } \\
\hline FAERS-only cases & 2 \\
\hline Literature-only cases & 9 \\
\hline FAERS and literature case overlap ${ }^{\mathrm{a}}$ & 3 \\
\hline \multicolumn{2}{|c|}{ Year received by FDA (initial) or published } \\
\hline 1995 & 2 \\
\hline 2004 & 4 \\
\hline 2011-2012 & 4 \\
\hline 2014-2018 & 4 \\
\hline \multicolumn{2}{|l|}{ Sex } \\
\hline Female & 7 \\
\hline Male & 7 \\
\hline \multicolumn{2}{|l|}{ Age in years } \\
\hline Mean & 53.5 \\
\hline Median & 47.5 \\
\hline Range & $33-70$ \\
\hline \multicolumn{2}{|l|}{ Country } \\
\hline Non-USA & 12 \\
\hline USA & 2 \\
\hline \multicolumn{2}{|l|}{ Ketamine route } \\
\hline Intravenous infusion & 10 \\
\hline Oral & 2 \\
\hline Not reported & 2 \\
\hline \multicolumn{2}{|l|}{ Reason for ketamine use } \\
\hline Complex regional pain syndrome & 9 \\
\hline Postherpetic neuralgia & 2 \\
\hline Chronic migraines & 1 \\
\hline Chronic pain unspecified & 1 \\
\hline Chronic facial pain & 1 \\
\hline \multicolumn{2}{|l|}{ Adverse event stratified by time to onset ${ }^{\mathrm{b}}$} \\
\hline $6 \mathrm{~h}$ (cycle 2) & 1 \\
\hline 1-4 days & 9 \\
\hline $1-2$ weeks & 3 \\
\hline $1-2$ months & 1 \\
\hline 10 years & 1 \\
\hline Time to onset not reported & 6 \\
\hline \multicolumn{2}{|c|}{ Characteristics of hepatobiliary events per case ${ }^{c}$} \\
\hline Elevated ALT & 10 \\
\hline Elevated GGT & 8 \\
\hline Elevated AST & 8 \\
\hline Elevated ALP & 8 \\
\hline Elevated total bilirubin levels & 5 \\
\hline Elevated unspecified liver enzymes & 4 \\
\hline Elevated direct bilirubin levels & 2 \\
\hline Biliary dilation & 2 \\
\hline Jaundice & 2 \\
\hline Liver cirrhosis & 1 \\
\hline Pericholeductal fibrosis & 1 \\
\hline Cholangitis & 1 \\
\hline
\end{tabular}

Table 1 (continued)

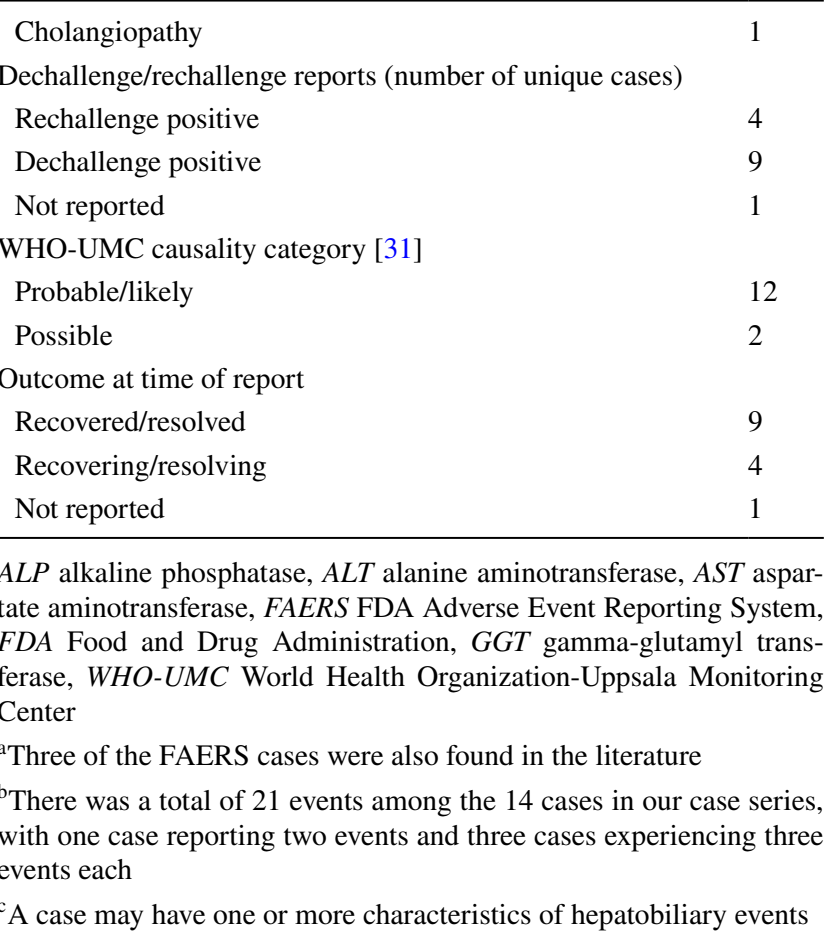

exclude possible viral etiologies; however, the remaining cases did not. Alcohol consumption was addressed in only three cases with two reporting no history, and one reporting occasional use. We did not identify cases with an outcome of fulminant hepatic necrosis, liver transplant, or death.

\subsection{Representative FAERS Case of Ketamine-Associated Hepatobiliary Adverse Events}

A 46-year-old female patient with chronic pain experienced elevated liver enzymes following a ketamine intravenous infusion. ${ }^{9}$ Past medical history included ongoing adrenal suppression, seronegative spondyloarthropathy, complex pain, anterior uveitis, reduced left ventricular function, type 2 diabetes mellitus, depression, and atonic bladder. Concomitant medications included clotrimazole, duloxetine, gabapentin, hypromellose, sulfasalazine,${ }^{10}$ clonazepam, haloperidol, calcium carbonate, buprenorphine, bisoprolol, atorvastatin ${ }^{j}$, furosemide, tramadol, paracetamol, hydromorphone, omeprazole, enalapril ${ }^{\mathrm{j}}$, mirtazapine, insulin aspart, ferrous sulfate, metformin hydrochloride, and prednisolone. A ketamine intravenous infusion was initiated at a rate of

\footnotetext{
9 This case has not been previously reported in the medical literature. ${ }^{10}$ Among the list of ongoing concomitant drugs, the report states that sulfasalazine, atorvastatin, and enalapril were "held".
} 


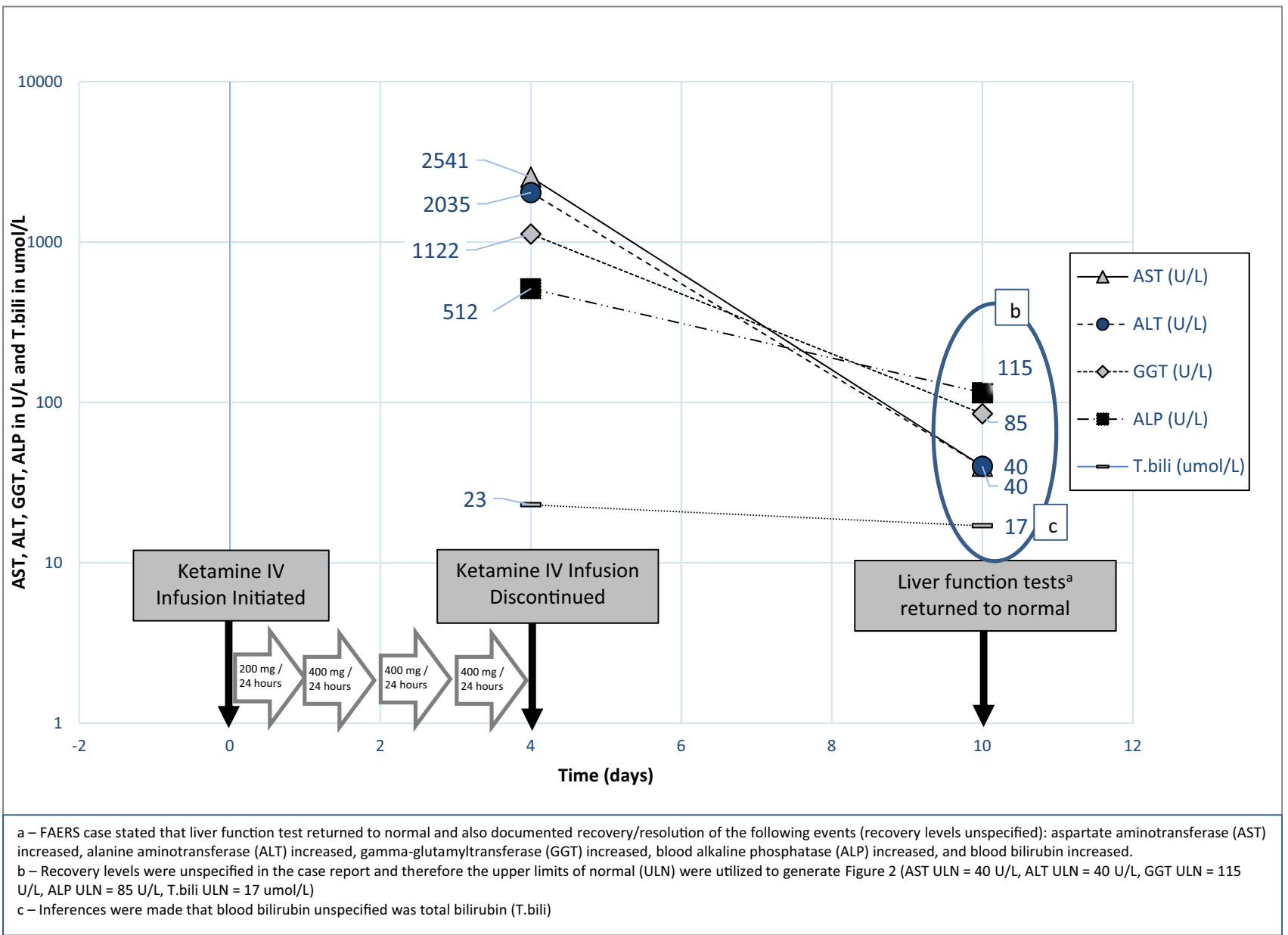

Fig. 2 FDA Adverse Event Reporting System (FAERS) case of 'transaminitis' 4 days following a ketamine infusion with recovery following discontinuation. $I V$ intravenous. (a) FAERS case stated that liver function test returned to normal and also documented recovery/resolution of the following events (recovery levels unspecified): aspartate aminotransferase (AST) increased, alanine aminotransferase (ALT) increased, gamma-glutamyl transferase (GGT) increased,

$200 \mathrm{mg} / 24$ hours for $24 \mathrm{~h}$, and then increased to $400 \mathrm{mg} / 24$ hours for 3 days for chronic pain. On the fourth day of the ketamine infusion, the patient experienced transaminitis with the following laboratory values: alanine aminotransferase $2035 \mathrm{U} / \mathrm{L}(\mathrm{ULN}=40 \mathrm{U} / \mathrm{L})$, aspartate aminotransferase $2541 \mathrm{U} / \mathrm{L}(\mathrm{ULN}=35 \mathrm{U} / \mathrm{L})$, ALP $512 \mathrm{U} / \mathrm{L}(\mathrm{ULN}=$ $115 \mathrm{U} / \mathrm{L})$, bilirubin $23 \mathrm{umol} / \mathrm{L}(\mathrm{ULN}=17.1 \mathrm{umol} / \mathrm{L})$, and gamma-glutamyl transferase $1122 \mathrm{U} / \mathrm{L}(\mathrm{ULN}=85 \mathrm{U} / \mathrm{L})$. Ketamine was permanently withdrawn, and liver function tests returned to normal 6 days after ketamine withdrawal (Fig. 2). blood alkaline phosphatase (ALP) increased, and blood bilirubin increased. (b) Recovery levels were unspecified in the case report and therefore the upper limits of normal (ULN) were utilized to generate Figure $2($ AST ULN $=40 \mathrm{U} / \mathrm{L}$, ALT ULN $=40 \mathrm{U} / \mathrm{L}$, GGT ULN $=115 \mathrm{U} / \mathrm{L}$, ALP ULN $=85 \mathrm{U} / \mathrm{L}$, T.bili ULN $=17 \mathrm{umol} / \mathrm{L})(\mathrm{c})$ Inferences were made that blood bilirubin unspecified was total bilirubin (T.bili)

\subsection{Ketamine Use Trends}

IQVIA NSP data provided a national estimate of ketamine sold from manufacturers to various channels of distribution. Sales of ketamine vials increased from 1.2 million vials in 2013 to 2.1 million vials in 2017 (Fig. 3). In 2017, 54\% of these vials were sold to non-federal hospitals, $37 \%$ directly to clinics, and $9 \%$ to other distribution channels (e.g., federal facilities, long-term care, mail-order, and chain/independent pharmacies). Although manufacturer sales of ketamine to all channels of distribution increased, the increase was 
Fig. 3 Nationally estimated number of ketamine vials sold by manufacturers, stratified by US channels of distribution. ${ }^{\dagger}$ Clinics include but are not limited to dialysis, family planning, $\mathrm{x}$-ray, oncology, emergicenters, and surgicenters. Veterinary clinics not included. ${ }^{\dagger}$ All other channels include but are not limited to federal facilities, long-term care, mail-order, and chain/independent pharmacies. Source: IQVIA, National Sales Perspectives ${ }^{\mathrm{TM}}$ (NSP). Years 2013-2017. Extracted July 2018.
2.5

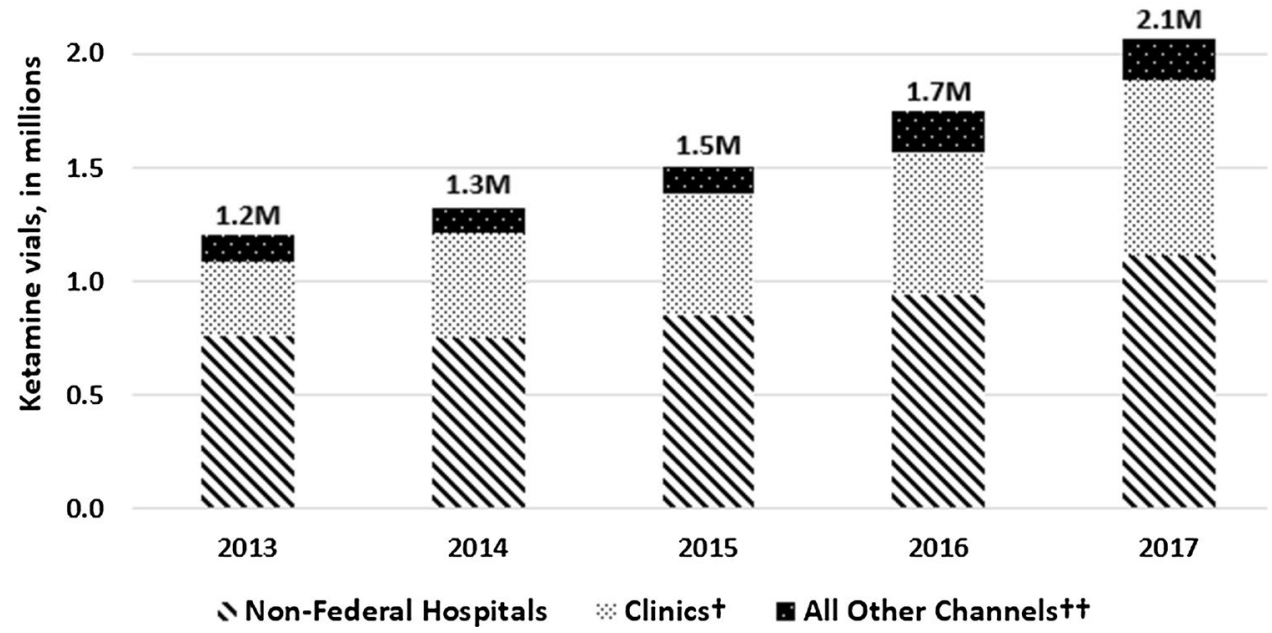

most notable in clinic settings, where the number of vials sold more than doubled in the 5 years examined from an estimated 332,000 sold in 2013 to 765,500 sold in 2017. These sales data describe product distribution but do not provide information on the indication of use for these products, thus we could not discern reasons for ketamine use in these ambulatory settings. National data on indication of use directly linked with the administration of ketamine in the clinic or hospital settings were not available for review.

\section{Discussion}

This analysis of FAERS and literature reports represents the largest published case series to date focused on hepatobiliary AEs arising from repeated or continuous ketamine exposure in the context of medical use. Twelve of the 14 cases that we identified demonstrated a 'probable' causal association with ketamine, using WHO-UMC system causality criteria. A causal relationship between ketamine and hepatobiliary AEs is inferred in individual cases from the clinical signature, temporal association with drug exposure, and diagnostic exclusion of alternative causes. Identified hepatobiliary AEs ranged from a reversible asymptomatic elevation in both serum liver aminotransferases and ALP to the development of permanent structural changes in the liver including cirrhosis or pericholeductal fibrosis. These findings highlight important parallels experienced by patients receiving ketamine for off-label medical use and those individuals abusing ketamine [19, 34, 37-40]. In a recent publication, Mallet et al. documented the findings of cholangiopathy in five patients with Coronavirus Infectious Disease 2019 (COVID-19) exposed to intravenous ketamine while mechanically ventilated [41]. The authors noted that liver injury was dose dependent and progressive, and total ketamine exposure correlated with outcome, including liverrelated death [41]. Ketamine is approved for diagnostic and surgical procedures, and for the induction of anesthesia. Our data support growing use of this product over the period 2013-2017, including in ambulatory settings.

Findings in this case series are consistent with results from prior studies showing potential hepatobiliary toxicity associated with recurrent use of ketamine [7, 17-22, 42]. Animal studies performed in mice treated long term with ketamine showed fatty degeneration of liver cells, fibrosis, and a rise in liver enzymes [43]. Alcohol increased the severity of these effects in mice, suggesting the importance of other potential risk factors, as identified in our case series. The exact mechanism for hepatobiliary injury is unknown [44], but hypothesized to be due to a reduction in hepatic oxygen delivery, and increased lipid peroxidation with free radical formation [45]. As an anesthetic agent, ketamine has been shown to cause transient abnormalities in serum liver tests [42]. Additionally, there are numerous reports of reversible cholestatic injury occurring in the context of ketamine abuse [40]. One case series from the Hong Kong Poison Information Centre reviewed emergency department medical records of 233 individuals abusing ketamine. Of the 178 with laboratory tests, $16 \%$ had abnormal serum liver tests, marked by rises of ALP from 100 to 508 U/L (reference range 35-98 U/L) and alanine aminotransferase from 52 to 180 U/L (reference level < 41 U/L) [20].

Hepatobiliary injury associated with ketamine may have important implications for long-term esketamine use in depression. In March 2019, the FDA approved esketamine for treatment-resistant depression in conjunction with an oral antidepressant [9]. In the clinical development program of 1709 patients exposed to esketamine, the FDA observed no clinically significant hepatobiliary AEs. Because clinical trials have strict inclusion and exclusion criteria, and 
measure efficacy and safety after a limited duration of exposure, AEs may not manifest until there is sufficient use in a wider patient population under real-world conditions. The FDA will closely monitor available postmarketing data for clinically significant hepatobiliary AEs associated with esketamine.

We identified hepatobiliary toxicity that ranges in severity from mild asymptomatic liver injury to severe symptomatic liver disease. All cases reported an increase in one or more serum liver tests, whereas the most severe cases reported biliary dilation, jaundice, liver cirrhosis, pericholeductal fibrosis, cholangitis, and cholangiopathy. In nine cases providing an alanine aminotransferase and ALP level, the pattern of liver injury was categorized as cholestatic $(n=3)$, mixed $(n$ $=3$ ), and hepatocellular ( $n=3$ ) based on the $R$ value [46]. We did not identify any cases of fulminant hepatic necrosis, liver transplant, or death. In some cases, we found notable evidence of a probable or likely causal association between repeated ketamine use and hepatobiliary events such as positive dechallenge and rechallenge responses to discontinuation and reintroduction of ketamine, respectively.

Sales data represent the amount of product sold from manufacturers to various settings of care and do not necessarily reflect what is being administered to patients directly. We evaluated sales distribution data to provide a national estimate of the number of ketamine vials sold from manufacturers into all channels of distribution. Although sales data do not provide a direct measure of patient use, the amount of product purchased by various settings of care is a reasonable surrogate for use, if we assume that facilities purchase drugs in quantities reflective of actual patient use. Based on national sales distribution patterns of ketamine, utilization appears to be largely in the hospital setting, likely because of the approved indications as an anesthetic agent [1]. However, it appears sales of ketamine to clinic settings have doubled in the 5 years examined, suggesting changes in utilization patterns. A web-based survey of US physicians showed a mix of specialists in a variety of geographic locations providing ketamine-based treatment for unapproved indications [7]. Although the survey has limited generalizability given the small size, it helps provide some insight into the evolving uses of ketamine [7]. This survey highlights two phenomena: (1) providers outside the realm of anesthesiology are using ketamine and (2) providers outside the realm of psychiatry are providing ketamine to treat a wide spectrum of pain syndromes and mental illness. Our findings with sales data, and recent drug approval of esketamine nasal spray for the treatment of treatment-resistant depression in adults [9], underscore a growing need to monitor the risks of repeated or long-term exposure to ketamine.

We highlight several limitations in our analysis. With FAERS data, as with other spontaneous reporting systems used in pharmacovigilance, the ability to make an inference whether a causal relationship exists between a specific drug and an AE may be limited because of variable report quality and the presence of confounders. In unfiltered FAERS reports, there is no a priori certainty that the reported events are actually due to the suspect product. In addition, the FDA does not require that a causal relationship between a product and event be proven, and reports do not always contain enough detail to properly evaluate an event. As such, cases ordinarily submitted to FAERS merely establish an association between a suspect drug or biological agent and the AE(s) described. Further, as a denominator is not available in spontaneous reporting systems, we cannot estimate the absolute risk of an $\mathrm{AE}$ occurring, which would be better addressed in prospective long-term observational studies. Despite these limitations, the phenotype of cholestatic injury with narrow and/or dilated bile duct segments seen in a portion of the cases in this series has been previously causally associated with repeated misuse and abuse of insufflated and parenteral formulations of ketamine [17, 18, 40]. Moreover, the recurrence of serum biochemical abnormalities after re-challenge with ketamine and rapid reversal of these changes upon discontinuation of this agent in some of the case reports is a strong indicator of its probable causal association with the reported AE.

\section{Conclusions}

We report an association between ketamine and a range of hepatobiliary events in the context of medically supervised, off-label, repeated, or continuous use. Ketamine product labeling was updated in August 2020 to convey the potential risk of hepatobiliary dysfunction with recurrent use [1]. Still, many uncertainties remain regarding the safety and effectiveness of off-label, medically supervised, repeated ketamine use. Prospective cohort trials and epidemiologic studies could clarify remaining questions, such as at-risk populations, cumulative dose risks, risks specific to certain routes of administration, and strategies to monitor and mitigate AEs.

This case series is based on contributions from the medical community. Voluntary reporting of AEs associated with drug products, by healthcare professionals and patients, is an important activity to support the safe use of FDA-approved drug therapies. We encourage continued reporting of drugrelated AEs through MedWatch: the FDA Safety Information and Adverse Event Reporting Program (http://www.fda.gov/ Safety/MedWatch/default.htm).

Supplementary Information The online version contains supplementary material available at https://doi.org/10.1007/s40264-021-01120-9. 


\section{Declarations}

Funding Not applicable.

Conflict of interest Samantha Cotter, Jennie Wong, Neha Gada, Rajdeep Gill, S. Christopher Jones, Grace Chai, Daniel Foster, Mark Avigan, and Mallika Mundkur declare that they have no conflict of interest.

Ethics approval Not applicable.

Consent to participate Not applicable.

Consent for publication Not applicable.

Availability of data and material Not applicable.

Code availability Not applicable.

Author contributions All authors contributed to the study conception and design. Material preparation, data collection, and analysis were performed by Samantha Cotter, Jennie Wong, Neha Gada, Rajdeep Gill, S. Christopher Jones, Grace Chai, Daniel Foster, Mark Avigan, and Mallika Mundkur. The first draft of the manuscript was written by Samantha Cotter and all authors commented on previous versions of the manuscript. All authors read and approved the final manuscript.

\section{References}

1. Ketalar (ketamine hydrochloride) [package insert]. Chestnut Ridge: Par Pharmaceutical; 2020.

2. Berman RM, Cappiello A, Anand A, et al. Antidepressant effects of ketamine in depressed patients. Biol Psychiatry. 2000;47(4):351-4.

3. Hocking G, Cousins MJ. Ketamine in chronic pain management: an evidence-based review. Anesth Analg. 2003;97(6):1730-9.

4. Zarate CA, Singh JB, Carlson PJ, et al. A randomized trial of an $N$-methyl-D-aspartate antagonist in treatment-resistant major depression. Arch Gen Psychiatry. 2006;63(8):856-64.

5. Schwartzman RJ, Alexander GM, Grothusen JR, Paylor T, Reichenberger E, Perreault M. Outpatient intravenous ketamine for the treatment of complex regional pain syndrome: a doubleblind placebo controlled study. Pain. 2009;147(1-3):107-15.

6. Sigtermans MJ, van Hilten JJ, Bauer MC, et al. Ketamine produces effective and long-term pain relief in patients with complex regional pain syndrome type 1. Pain. 2009;145(3):304-11.

7. Wilkinson ST, Toprak M, Turner MS, Levine SP, Katz RB, Sanacora G. A survey of the clinical, off-label use of ketamine as a treatment for psychiatric disorders. Am J Psychiatry. 2017;174(7):695-6.

8. Andrade C. Ketamine for depression, 4: in what dose, at what rate, by what route, for how long, and at what frequency? J Clin Psychiatry. 2017;78(7):e852-7.

9. Spravato (esketamine) [prescribing information]. Titusville: Janssen Pharmaceuticals, Inc.; 2020.

10. Sanacora G, Frye MA, McDonald W, et al. A consensus statement on the use of ketamine in the treatment of mood disorders. JAMA Psychiat. 2017;74(4):399-405.

11. Zhao J, Wang Y, Wang D. The effect of ketamine infusion in the treatment of complex regional pain syndrome: a systemic review and meta-analysis. Curr Pain Headache Rep. 2018;22(2):12.

12. Cohen SP, Bhatia A, Buvanendran A, et al. Consensus guidelines on the use of intravenous ketamine infusions for chronic pain from the American Society of Regional Anesthesia and Pain Medicine, the American Academy of Pain Medicine, and the American Society of Anesthesiologists. Reg Anesth Pain Med. 2018;43(5):521-46.

13. Noppers I, Niesters M, Aarts L, Smith T, Sarton E, Dahan A. Ketamine for the treatment of chronic non-cancer pain. Expert Opin Pharmacother. 2010;11(14):2417-29.

14. Szymkowicz SM, Finnegan N, Dale RM. A 12-month naturalistic observation of three patients receiving repeat intravenous ketamine infusions for their treatment-resistant depression. J Affect Disord. 2013;147(1-3):416-20.

15. Quibell R, Fallon M, Mihalyo M, Twycross R, Wilcock A. Ketamine. J Pain Symptom Manag. 2015;50(2):268-78.

16. Wilkinson ST, Sanacora G. Considerations on the off-label use of ketamine as a treatment for mood disorders. JAMA. 2017;318(9):793-4.

17. Gutkin E, Hussain SA, Kim SH. Ketamine-induced biliary dilatation: from Hong Kong to New York. J Addict Med. 2012;6(1):89-91.

18. Wong SW, Lee KF, Wong J, Ng WW, Cheung YS, Lai PB. Dilated common bile ducts mimicking choledochal cysts in ketamine abusers. Hong Kong Med J. 2009;15(1):53-6.

19. Lo RS, Krishnamoorthy R, Freeman JG, Austin AS. Cholestasis and biliary dilatation associated with chronic ketamine abuse: a case series. Singapore Med J. 2011;52(3):e52-5.

20. Ng SH, Tse ML, Ng HW, Lau FL. Emergency department presentation of ketamine abusers in Hong Kong: a review of 233 cases. Hong Kong Med J. 2010;16(1):6-11.

21. Wang JW, Kivovich V, Gordon L. Ketamine abuse syndrome: hepatobiliary and urinary pathology among adolescents in Flushing, NY. Pediatr Emerg Care. 2017;33(8):e24-6.

22. Yu WL, Cho CC, Lung PF, et al. Ketamine-related cholangiopathy: a retrospective study on clinical and imaging findings. Abdom Imaging. 2014;39(6):1241-6.

23. Li L, Vlisides PE. Ketamine: 50 years of modulating the mind. Front Hum Neurosci. 2016;10:612.

24. Agence Nationale de Sécurité du Médicament et des Produits de Sánte. Kétamine: risque d'atteintes hépatiques graves lors d'utilisations prolongées et/ou à doses élevées: lettre aux professionnels de santé. Updated 2017. https://ansm.sante.fr/S-informer/ Informations-de-securite-Lettres-aux-professionnels-de-sante/ Ketamine-risque-d-atteintes-hepatiques-graves-lors-d-utilisatio ns-prolongees-et-ou-a-doses-elevees-Lettre-aux-professionnelsde-sante. Accessed 22 Jan 2021.

25. National Pharmaceutical Regulatory Agency (NPRA), Malaysia. Ketamine: risk of severe liver damage with repeated and/or prolonged use at high doses. Updated June 2017. https://www.npra. gov.my/images/Publications/REAKSI_Drug_Safety_News/2017/ REAKSI62017.pdf. Accessed 22 Jan 2021.

26. MedDRA MSSO. Introductory guide for Standardized MedDRA Queries (SMQs). Version 21.0. Updated March 2018. https:// admin.new.meddra.org/sites/default/files/guidance/file/smq_intgu ide_21_0_english.pdf. Accessed 22 Jan 2021.

27. LiverTox: clinical and research information on drug-induced liver injury. Bethesda (MD): National Institute of Diabetes and Digestive and Kidney Diseases; 2012. Severity grading in drug induced liver injury. Updated 4 May 2019. https://www.ncbi.nlm.nih.gov/ books/NBK548241/. Accessed 24 June 2021.

28. Nicoll, D. Appendix: therapeutic drug monitoring and laboratory reference ranges. In: McPhee S, Papadakis M, editors. Current medical diagnosis and treatment. 46th ed. Columbus: McGraw Hill; 2007. p. 1767-75.

29. Correll GE, Maleki J, Gracely EJ, Muir JJ, Harbut RE. Subanesthetic ketamine infusion therapy: a retrospective analysis of a novel therapeutic approach to complex regional pain syndrome. Pain Med. 2004;5(3):263-75. 
30. National Institute on Drug Abuse. Health consequences of drug misuse. Introduction. Updated June 9, 2020. https://www.druga buse.gov/drug-topics/health-consequences-drug-misuse/introducti on. Accessed 22 Jan 2021.

31. World Health Organization (WHO)-Uppsala Monitoring Centre. The use of the WHO-UMC system for standardized case causality assessment. Updated June 2013. https://www.who.int/publicatio ns/m/item/WHO-causality-assessment. Accessed 22 Jan 2021.

32. LiverTox: clinical and research information on drug-induced liver injury. Bethesda (MD): National Institute of Diabetes and Digestive and Kidney Diseases; 2012. Updated 21 January, 2021. https://www.ncbi.nlm.nih.gov/books/NBK547852/?report=class ic. Accessed 22 Jan 2021.

33. Bevan R, Burke D. Ketamine as a possible cause of cirrhosis in a patient with chronic pain. Frontline Gastroenterol. 2014;5(3):208-10.

34. Tofani CJ, Halegoua-DeMarzio D. BLOG: 58 year old female with acute liver injury related to IV ketamine treatment for migraines. Healio Gastroenterology. January 2016. https://www.healio.com/ news/gastroenterology/20160105/blog-58-year-old-female-withacute-liver-injury-related-to-intermittent-iv-ketamine-for-treat ment. Accessed 22 Jan 2021.

35. Tofani C, Gandhi K, Halegoua-DeMarzio D. Acute liver injury related to intermittent intravenous ketamine for the treatment of migraines: 765. Am Coll Gastroenterol ACG. 2015;110:S334-5.

36. Hewitt NA, Cox P. Recurrent subanesthetic ketamine infusions for complex regional pain syndrome leading to biliary dilation, jaundice, and cholangitis: a case report. A A Pract. 2018;10(7):168-70.

37. Kato Y, Homma I, Ichiyanagi K. Postherpetic neuralgia. Clin J Pain. 1995;11(4):336-8.
38. Noppers IM, Niesters M, Aarts LP, et al. Drug-induced liver injury following a repeated course of ketamine treatment for chronic pain in CRPS type 1 patients: a report of 3 cases. Pain. 2011;152(9):2173-8.

39. Turkish A, Luo JJ, Lefkowitch JH. Ketamine abuse, biliary tract disease, and secondary sclerosing cholangitis. Hepatology. 2013;58(2):825-7.

40. Wong GL, Tam $\mathrm{YH}, \mathrm{Ng} \mathrm{CF}$, et al. Liver injury is common among chronic abusers of ketamine. Clin Gastroenterol Hepatol. 2014;12(10):1759-62.e1.

41. Mallet V, Bock K, Doumbe Mandengue P, Dufour N, et al. Intravenous ketamine and progressive cholangiopathy in COVID-19 patients. J Hepatol. 2021;74:1243-4. https://doi.org/10.1016/j. jhep.2021.02.007.

42. LiverTox: clinical and research information on drug-induced liver injury. Bethesda (MD): National Institute of Diabetes and Digestive and Kidney Diseases; 2012. Ketamine. Updated April 25, 2018. https:// www.ncbi.nlm.nih.gov/books/NBK548337/. Accessed 22 Jan 2021.

43. Wai MS, Chan WM, Zhang AQ, Wu Y, Yew DT. Long-term ketamine and ketamine plus alcohol treatments produced damages in liver and kidney. Hum Exp Toxicol. 2012;31(9):877-86.

44. Dundee JW, Fee JP, Moore J, McIlroy PD, Wilson DB. Changes in serum enzyme levels following ketamine infusions. Anaesthesia. 1980;35(1):12-6.

45. Sear JW. Ketamine hepato-toxicity in chronic pain management: another example of unexpected toxicity or a predicted result from previous clinical and pre-clinical data? Pain. 2011;152(9):1946-7.

46. Fontana RJ, Seeff LB, Andrade RJ, et al. Standardization of nomenclature and causality assessment in drug-induced liver injury: summary of a clinical research workshop. Hepatology. 2010;52(2):730-42. 